\title{
From the Beginning of the World to the Beginning of Life on Earth
}

\author{
Vladimir S. Netchitailo \\ Biolase Inc., Irvine, USA \\ Email:netchitailov@gmail.com
}

How to cite this paper: Netchitailo, V.S (2021) From the Beginning of the World to the Beginning of Life on Earth. Journal of High Energy Physics, Gravitation and Cosmology, 7, 1503-1523.

https://doi.org/10.4236/jhepgc.2021.74092

Received: September 28, 2021

Accepted: October 23, 2021

Published: October 26, 2021

Copyright $\odot 2021$ by author(s) and Scientific Research Publishing Inc. This work is licensed under the Creative Commons Attribution International License (CC BY 4.0).

http://creativecommons.org/licenses/by/4.0/

(c) (i) Open Access

\begin{abstract}
Hypersphere World-Universe Model (WUM) is, in fact, a Paradigm Shift in Cosmology [1]. In this paper, we provide seven Pillars of WUM: Medium of the World; Inter-Connectivity of Primary Cosmological Parameters; Creation of Matter; Multicomponent Dark Matter; Macroobjects; Volcanic Rotational Fission; Dark Matter Reactors. We describe the evolution of the World from the Beginning up to the birth of the Solar System and discuss the condition of the Early Earth before the beginning of life on it.
\end{abstract}

\section{Keywords}

Hypersphere World-Universe Model, Law of Conservation of Angular Momentum, Dark Epoch, Volcanic Rotational Fission, Luminous Epoch, Dark Matter Particles, Macroobject Shell Model, Dark Matter Core, Medium of the World, Dark Matter Fermi Bubbles, Galactic Wind, Solar Wind, Intergalactic Plasma, Macroobjects, Gravitomagnetic Parameter, Impedance, Energy Density, Gravitational Parameter, Hubble's Parameter, Temperature of Microwave Background Radiation, Inter-Connectivity of Primary Cosmological Parameters, Dark Matter Reactor, Early Earth, Formation of Earth, Origin of Moon, Continental Crust of Earth, Earth's Atmosphere and Oceans, Origin of Life

\section{Introduction}

Hypersphere World-Universe Model (WUM) was developed for the last 20 years and is, in fact, a Paradigm Shift in Cosmology [1]. The seven Pillars of WUM are as follows:

- Medium of the World;

- Inter-Connectivity of Primary Cosmological Parameters;

- Creation of Matter; 
- Multicomponent Dark Matter;

- Macroobjects;

- Volcanic Rotational Fission;

- Dark Matter Reactors.

Cosmology is a branch of Classical Physics. It should then be described by classical notions, which define emergent phenomena. By definition, an emergent phenomenon is a property that is a result of simple interactions that work cooperatively to create a more complex interaction. Physically, simple interactions occur at microscopic level, and the collective result can be observed at macroscopic level.

\section{Medium of the World}

Physical Aether was suggested as early as the $17^{\text {th }}$ century, by I. Newton. Following the work of T. Young (1804) and A. J. Fresnel (1816), it was believed that light propagates as a transverse wave within an elastic medium called Luminiferous Aether, which was abandoned in 1905. In later years there have been classical physicists who advocated the existence of Aether [2]:

- N. Tesla declared in 1937: All attempts to explain the workings of the universe without recognizing the existence of the Aether and the indispensable function it plays in the phenomena are futile and destined to oblivion [3];

- P. Dirac stated in 1951 in an article "Is there an Aether?" that we are rather forced to have an Aether [4].

WUM introduces the Medium of the World, which consists of stable elementary particles with lifetimes longer than the age of the World: protons, electrons, photons, neutrinos, and Dark Matter Particles (DMPs). The existence of the Medium is a principal point of WUM. It follows from the observations of Intergalactic Plasma; Cosmic Microwave Background Radiation; Far-Infrared Background Radiation. Inter-galactic voids discussed by astronomers are, in fact, examples of the Medium in its purest. Cosmic Microwave Background Radiation is part of the Medium; it then follows that the Medium is the absolute frame of reference. Relative to the Cosmic Microwave Background rest frame, the Milky Way galaxy and the Sun are moving with the speed of 552 and $370 \mathrm{~km} \cdot \mathrm{s}^{-1}$, respectively [5].

The energy density of the Medium is $2 / 3$ of the total energy density of the World. Superclusters, Galaxies, Extrasolar systems, planets, moons, etc. are made of the same particles. The energy density of Macroobjects adds up to $1 / 3$ of the total energy density of the World throughout the World's evolution [5]. Cosmological Principal is valid for the Homogeneous and Isotropic Medium. The distribution of Macroobjects is Inhomogeneous and Anisotropic, and therefore, the Cosmological Principal is not viable for the entire World.

WUM is the classical model, therefore classical notions can be introduced only when the very first ensemble of particles was created at the cosmological time $\tau_{M}$ equals to: $\tau_{M}=\alpha^{-2} \times t_{0} \cong 10^{-18} \mathrm{~s}$, where $\alpha$ is the dimensionless Rydberg 
constant: $\alpha=\left(2 a R_{\infty}\right)^{1 / 3}$ (that was later named "Fine-structure constant"); $t_{0}$ is a basic unit of time: $t_{0}=a / c=5.9059662 \times 10^{-23} \mathrm{~s}$; $a$ is a basic unit of size $a=1.7705641 \times 10^{-14} \mathrm{~m}$ and $c$ is a gravitodynamic constant. It is worth noting that the speed of light in vacuum, commonly denoted as $c$, is not related to the World in our Model, because there is no vacuum in it. Instead, there is the Medium of the World consisting of elementary particles. In WUM, the cosmological principal Universality of Physical Laws is valid at the cosmological times $\tau \geq \tau_{M}$ because the Laws are determined by the Medium of the World.

In frames of WUM, Time and Space are closely connected with the Mediums' impedance (wave resistance) $Z_{g}$ that equals to the Hubble's parameter $H$ : $Z_{g}=H=\tau^{-1}$ and the gravitomagnetic parameter $\mu_{g}$, which equals to: $\mu_{g}=R^{-1}$. It follows that neither Time nor Space could be discussed in absence of the Medium. The gravitational parameter $G$ that is proportional to the Mediums' energy density can be introduced only for the Medium filled with Matter. The Gravitation is a result of simple interactions of DMPs with Matter (by the introduced new Weak Interaction) that work cooperatively to create a more complex interaction. DMPs are responsible for Le Sage's mechanism of the gravitation. Gravity, Space and Time are all emergent phenomena [5]. In this regard, it is worth recalling Albert Einstein quote: "When forced to summarize the theory of relativity in one sentence: time and space and gravitation have no separate existence from matter".

\section{Inter-Connectivity of Primary Cosmological Parameters}

The constancy of the universe fundamental constants, including Newtonian constant of gravitation, is now commonly accepted, although has never been firmly established as a fact. All conclusions on the constancy of $G$ are model-dependent. A commonly held opinion states that gravity has no established relation to other fundamental forces, so it does not appear possible to calculate it from other constants that can be measured more accurately, as is done in some other areas of physics.

WUM holds that there indeed exist relations between all Primary Cosmological Parameters that depend on dimensionless time-varying quantity $Q$ that is a measure of the Size $R$ and Age $A_{t}$ of the World:

$$
Q=\frac{R}{a}=\frac{A_{\tau}}{t_{0}}
$$

which in present epoch equals to: $Q=0.759972 \times 10^{40}$. WUM is based on two parameters only: $\alpha$ and $Q$. The Model develops a mathematical framework that allows for direct calculation of the following parameters through $Q$ [6]:

- Newtonian parameter of gravitation $G$;

- Age of the World $A_{\tau}$;

- Size of the World $R$;

- Hubble's parameter $H$;

- Critical energy density $\rho_{c r}$; 
- Concentration of Intergalactic Plasma $n_{I G P}$;

- Minimum Energy of Photons $E_{p h}$;

- Temperature of the Microwave Background Radiation $T_{M B R}$;

- Temperature of the Far-Infrared Background Radiation peak $T_{\text {FIRB }}$;

- Fermi coupling parameter $G_{F}$;

- Electronic neutrino rest energy $E_{v_{e}}$;

- Muonic neutrino rest energy $E_{v_{\mu}}$;

- Tauonic neutrino rest energy $E_{v_{\tau}}$.

In frames of WUM, we calculate the values of these Primary Cosmological Parameters, which are in good agreement with the latest results of their measurements. For example:

- $\quad$ The predicted value of $G$ in 2013 [7]:

$$
G=6.674536 \times 10^{-11} \mathrm{~m}^{3} \cdot \mathrm{kg}^{-1} \cdot \mathrm{s}^{-2}
$$

is in excellent agreement with the experimentally measured by Qing Li, et al. in 2018 values using two independent methods [8]:

$$
\begin{aligned}
& G(1)=6.674184 \times 10^{-11} \mathrm{~m}^{3} \cdot \mathrm{kg}^{-1} \cdot \mathrm{s}^{-2}(11.64 \mathrm{ppm}) \\
& G(2)=6.67484 \times 10^{-11} \mathrm{~m}^{3} \cdot \mathrm{kg}^{-1} \cdot \mathrm{s}^{-2}(11.61 \mathrm{ppm})
\end{aligned}
$$

- The calculated value of $T_{M B R}=2.72518 \mathrm{~K}$ in the present epoch is in excellent agreement with experimentally measured value of $2.72548 \pm 0.00057 \mathrm{~K}$ [9]. It is worth noting that at the Beginning of the Luminous Epoch $(0.45$ Gyr) the calculated value was $T_{M B R}=6.4775 \mathrm{~K}$ and at the Birth of the Solar System (9.65 Gyr) $-T_{M B R}=3.0141 \mathrm{~K}$. Therefore, any Model describing creation of Macroobjects must hold true in cold World conditions;

- The Age of the World: $A_{\tau}=t_{0} \times Q=14.22 \mathrm{Gyr}$ is determined by the parameters of the Medium only.

In 2013, WUM revealed a self-consistent set of time-varying values of Primary Cosmological Parameters of the World, solved the Missing Baryon Problem and predicted the values of the following Cosmological parameters: Gravitation, Concentration of intergalactic plasma, and the Minimum energy of photons, which were experimentally confirmed in 2015-2020. "The Discovery of a Supermassive Compact Object at the Centre of Our Galaxy" (Nobel Prize in Physics 2020) made by R. Genzel and A. Ghez confirm one of the most important predictions of WUM in 2013: "Macroobjects of the World have cores made up of the discussed DM particles. Other particles, including DM and baryonic matter, form shells surrounding the cores" [10].

\section{Creation of Matter}

F. Hoyle and J. V. Narlikar in 1964 offered an explanation for the appearance of the new matter by postulating the existence of what they dubbed the "Creation field", or just the "C-field" [11]. P. Dirac in 1974 discussed a continuous creation of matter by an additive mechanism (uniformly throughout space) and a multiplicative mechanism (proportional to the amount of the existing matter) 
[12].

WUM follows the idea of the continuous creation of matter by the additive mechanism. To provide the creation of Matter by the Universe uniformly throughout the World, we consider the following Concept of the World proposed by G. Riemann in 1854 [13]: 3D Finite World is a Hypersphere of 4D Nucleus. In our view, the World was started by a Fluctuation in Eternal Universe, and 4D Nucleus of the World with a radius of a was born. The Nucleus is expanding in Its fourth spatial dimension and Its surface, the Hypersphere, is likewise expanding. The radius of the Nucleus $R$ is increasing with speed $c$ (gravitodynamic constant) for a cosmological time $\tau$ from the Beginning and equals to $R=c \tau$. By definition, the gravitodynamic constant $c$ is the ratio of the absolute gravitomagnetic unit of charge $E_{0}$ to the absolute gravitostatic unit of charge $E_{0} / c$, where $E_{0}$ is the basic unit of energy: $E_{0}=h c / a$ ( $h$ is the Planck constant).

The surface of the Nucleus is created in a process analogous to sublimation. Continuous creation of matter is the result of this process. Sublimation is a well-known endothermic process that happens when surfaces are intrinsically more energetically favorable than the bulk of a material, and hence there is a driving force for surfaces to be created.

Dark Matter (DM) is created by the Universe in the $4 \mathrm{D}$ Nucleus of the World. DMPs carry new DM into the 3D Hypersphere World. Ordinary Matter is a byproduct of DMPs self-annihilation. Consequently, a Matter-Antimatter Asymmetry Problem discussed in literature does not arise (since antimatter does not get created by DMPs self-annihilation). By analogy with 3D ball, which has $2 \mathrm{D}$ spherical surface (that has surface energy), we can imagine that the 3D Hypersphere World has a "Surface Energy" of the 4D Nucleus.

The proposed $4 \mathrm{D}$ process is responsible for the Expansion, Creation of Matter, and Arrow of Time. It constitutes the main Hypothesis of WUM. In our view, the arrow of the Cosmological Time does not depend on any physical phenomenon in the Medium of the World. It is the result of the Worlds' expansion due to the driving force for surfaces to be created [14]. It is important to emphasize that:

- Creation of Matter is a direct consequence of expansion;

- Creation of DM occurs homogeneously in all points of the 3D Hypersphere World.

\section{Multicomponent Dark Matter}

Two-component DM system consisting of bosonic and fermionic components is proposed for the explanation of emission lines from the bulge of Milky Way galaxy. C. Boehm, et al. analyze the possibility of two coannihilating neutral and stable DMPs: a heavy fermion for example, like the lightest neutralino ( $>100$ $\mathrm{GeV})$, and the other possibly a light spin-0 particle ( 100 MeV) [15].

WUM proposes multicomponent DM system consisting of two couples of coannihilating DMPs: a heavy Dark Matter Fermion (DMF)—DMF1 (1.3 TeV) 
and a light spin-0 boson-DIRAC (70 MeV) that is a dipole of Dirac's monopoles with charge $\mu=e / 2 \alpha$ ( $e$ is the elementary charge); a heavy fermion DMF2 $(9.6 \mathrm{GeV})$ and a light spin-0 boson-ELOP $(340 \mathrm{keV})$ that is a dipole of preons with electrical charge e/3; a self-annihilating fermion-DMF3 (3.7 keV), and a fermion DMF4 $(0.2 \mathrm{eV})$.

WUM postulates that rest energies of DMFs and bosons are proportional to the basic unit of energy $E_{0}$ multiplied by different exponents of $\alpha$ and can be expressed with the following formulae:

$\begin{array}{ll}\text { DMF1 (fermion): } & E_{D M F 1}=\alpha^{-2} E_{0}=1.3149950 \mathrm{TeV} \\ \text { DMF2 (fermion): } & E_{D M F 2}=\alpha^{-1} E_{0}=9.5959823 \mathrm{GeV} \\ \text { DIRAC (boson): } & E_{D I R A C}=\alpha^{0} E_{0}=70.025267 \mathrm{MeV} \\ \text { ELOP (boson): } & E_{E L O P}=2 / 3 \alpha^{1} E_{0}=340.66606 \mathrm{keV} \\ \text { DMF3 (fermion): } & E_{D M F 3}=\alpha^{2} E_{0}=3.7289402 \mathrm{keV} \\ \text { DMF4 (fermion): } & E_{D M F 4}=\alpha^{4} E_{0}=0.19857111 \mathrm{eV}\end{array}$

It is worth noting that the rest energy of electron $E_{e}$ equals to: $E_{e}=\alpha E_{0}$ and the Rydberg unit of energy is: $R y=h c R_{\infty}=0.5 \alpha^{3} E_{0}=13.605693 \mathrm{eV}$.

We still do not have a direct confirmation of DMPs' rest energies, but we do have a number of indirect observations. The signatures of DMPs self-annihilation with expected rest energies of $1.3 \mathrm{TeV}$; $9.6 \mathrm{GeV}$; $70 \mathrm{MeV}$; $340 \mathrm{keV}$; $3.7 \mathrm{keV}$ are found in spectra of the diffuse gamma-ray background and the emissions of various Macroobjects in the World. We connect observed gamma-ray spectra with the structure of Macroobjects (nuclei and shells composition). Self-annihilation of those DMPs can give rise to any combination of gamma-ray lines. Thus, the diversity of very high energy gamma-ray sources in the World has a clear explanation in WUM [16].

In this regard, it is worth recalling a story about neutrinos: "The neutrino was postulated first by W. Pauli in 1930 to explain how beta decay could conserve energy, momentum, and angular momentum (spin). But we still don't know the values of neutrino masses". Although we still cannot measure neutrinos' masses directly, no one doubts their existence.

\section{Macroobjects}

In WUM, Macrostructures of the World (Superclusters, Galaxies, Extrasolar systems) have Nuclei made up of DMFs, which are surrounded by Shells composed of DM and Baryonic Matter. The shells envelope one another, like a Russian doll. The lighter a particle, the greater the radius and the mass of its shell. Innermost shells are the smallest and are made up of heaviest particles; outer shells are larger and consist of lighter particles. Introduced principally new Weak Interaction of DMPs with Matter provides integrity of all shells: a distance between particles is smaller than the range of the weak interaction $R_{W}=1.65314 \times 10^{-4} \mathrm{~m}$ (see Section 7). Table 1 describes the parameters of Macroobjects Cores (which are Fermionic Compact Stars in WUM) in the present Epoch made up of different Fermions. 
Table 1. Parameters of Macroobjects cores made up of different fermions in present Epoch.

\begin{tabular}{ccccc}
\hline Fermion & $\begin{array}{c}\text { Fermion } \\
\text { Mass } \\
\boldsymbol{m}_{\boldsymbol{f}} \mathrm{MeV}\end{array}$ & $\begin{array}{c}\text { Macroobject } \\
\text { Mass } \\
\boldsymbol{M}_{\text {max }}, \mathbf{k g}\end{array}$ & $\begin{array}{c}\text { Macroobject } \\
\text { Radius } \\
\boldsymbol{R}_{\min }, \mathrm{m}\end{array}$ & $\begin{array}{c}\text { Macroobject } \\
\text { Density } \\
\boldsymbol{\rho}_{\text {max }}, \mathbf{k g m}^{-3}\end{array}$ \\
\hline DMF1 & $1.3 \times 10^{6}$ & $1.9 \times 10^{30}$ & $8.6 \times 10^{3}$ & $7.2 \times 10^{17}$ \\
DMF2 & $9.6 \times 10^{3}$ & $1.9 \times 10^{30}$ & $8.6 \times 10^{3}$ & $7.2 \times 10^{17}$ \\
Electron-Positron & 0.51 & $6.6 \times 10^{36}$ & $2.9 \times 10^{10}$ & $6.3 \times 10^{4}$ \\
DMF3 & $3.7 \times 10^{-3}$ & $1.2 \times 10^{41}$ & $5.4 \times 10^{14}$ & $1.8 \times 10^{-4}$ \\
DMF4 & $2 \times 10^{-7}$ & $4.2 \times 10^{49}$ & $1.9 \times 10^{23}$ & $1.5 \times 10^{-21}$ \\
\hline
\end{tabular}

The calculated parameters of the shells show that [5]:

- Nuclei made up of DMF1 and/or DMF2 compose Cores of stars in Extrasolar Systems;

- Shells of DMF3 and/or Electron-Positron plasma around Nuclei made up of DMF1 and/or DMF2 make up Cores of Galaxies;

- Nuclei made up of DMF1 and/or DMF2 surrounded by shells of DMF3 and DMF4 compose Cores of Superclusters.

According to WUM, Cores of Galaxies are DM Compact Objects made up of DMF1 and/or DMF2 with shell of DMF3 with the calculated maximum mass of $6 \times 10^{10} M_{\odot}$. This value is in good agreement with the experimentally obtained value of the most massive black hole ever found, with a mass of $6.6 \times 10^{10} M_{\odot}$ at the center of TON 618 [17]. It is worth noting that there are no black holes in WUM.

Laniakea Supercluster (LSC) is a galaxy supercluster that is home to Milky Way (MW) and approximately 100,000 other nearby galaxies (see Figure 1). It is known as one of the largest superclusters with estimated binding mass $10^{17} M_{\odot}$ [18] (see Table 2). The neighboring superclusters to LSC are the Shapley Supercluster, Hercules Supercluster, Coma Supercluster, and Perseus-Pisces Supercluster. Distance from the Earth to the Centre of LSC is 250 Mly, Redshift0.0708 (center).

We emphasize that about 100,000 nearby galaxies are moving around Centre of Laniakea Supercluster. They belong to LSC. All these galaxies did not start their movement from the "Initial Singularity". The neighboring superclusters have the same structure (see Figure 1 and Figure 2 in [1]). It means that the World is, in fact, a Multiworld consisting of $\gtrsim 10^{3}$ Superclusters (see Section 7). Big Bang never happened.

B. Carr, et al. "consider the observational constraints on stupendously large black holes (SLABS) in the mass range $M>10^{11} M_{\odot}$. These have attracted little attention hitherto, and we are aware of no published constraints on a SLAB population in the range $\left(10^{12}-10^{18}\right) M_{\odot}$. However, there is already evidence for black holes of up to nearly $10^{11} M_{\odot}$ in galactic nuclei [17], so it is conceivable that SLABs exist, and they may even have been seeded by primordial black holes" [21]. 


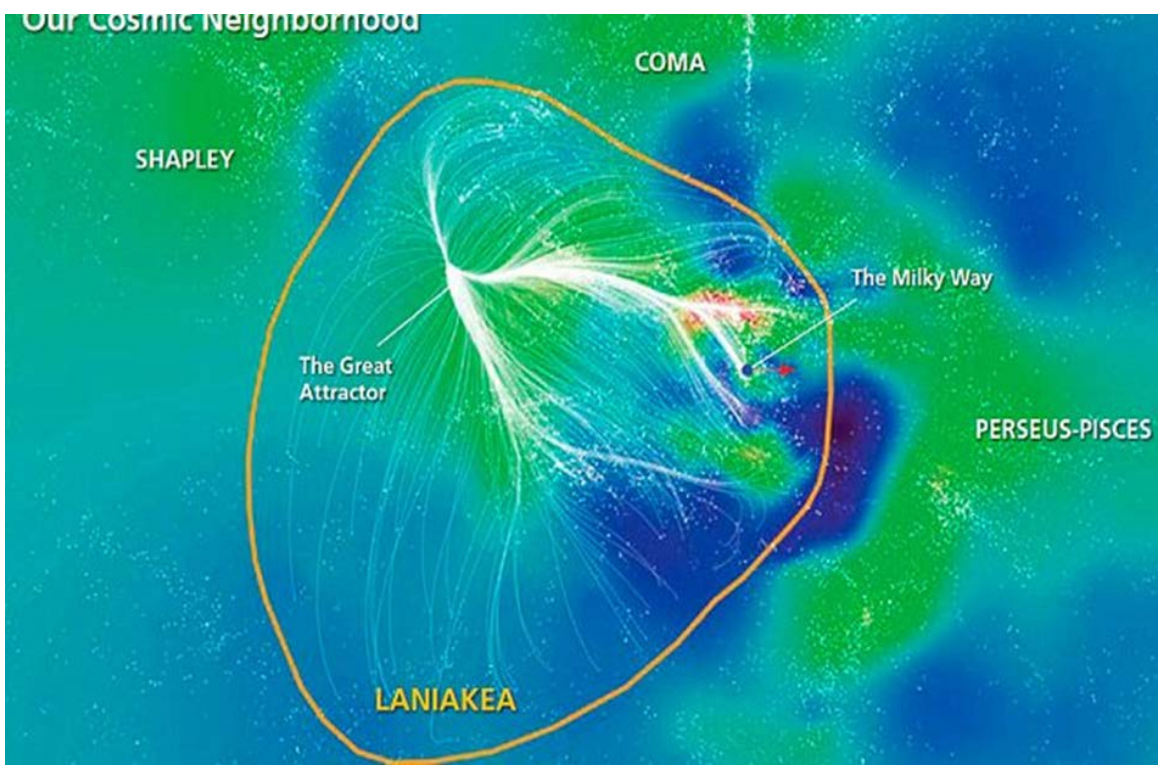

Figure 1. Laniakea Supercluster. Adapted from [19].

Table 2. Major axes of the largest superclusters. Adapted from [20].

\begin{tabular}{|c|c|c|}
\hline $\begin{array}{l}\text { Structure Name } \\
\text { (year discovered) }\end{array}$ & $\begin{array}{l}\text { Maximum Length } \\
\text { (in light years) }\end{array}$ & Note \\
\hline Caelum Supercluster & $910,000,000$ & $\begin{array}{c}\text { The Caelum Supercluster is a collection of over } \\
550,000 \text { galaxies. It is the largest galaxy } \\
\text { supercluster. }\end{array}$ \\
\hline Saraswati Supercluster & $652,000,000$ & $\begin{array}{l}\text { The Saraswati Supercluster consists of } 43 \text { massive } \\
\text { galaxy clusters, which include Abell } 2361 \text { and } \\
\text { ZWCl } 2341.1+0000 \text {. }\end{array}$ \\
\hline Boötes Supercluster & $620,000,000$ & \\
\hline $\begin{array}{l}\text { Horologium-Reticulum } \\
\text { Supercluster (2005) }\end{array}$ & $550,000,000$ & Also known as the Horologium Supercluster. \\
\hline Laniakea Supercluster (2014) & $520,000,000$ & Galaxy supercluster in which Earth is located. \\
\hline $\begin{array}{l}\text { Hyperion proto-supercluster } \\
\text { (2018) }\end{array}$ & $489,000,000$ & $\begin{array}{c}\text { The largest and earliest known } \\
\text { proto-supercluster. }\end{array}$ \\
\hline Draco Supercluster & $410,000,000$ & \\
\hline Great Attractor & $400,000,000$ & \\
\hline Shapley Supercluster & $400,000,000$ & $\begin{array}{c}\text { First identified by Harlow Shapley as a cloud of } \\
\text { galaxies in 1930, it was not identified as a } \\
\text { structure until } 1989 .\end{array}$ \\
\hline Virgo Supercluster & $110,000,000$ & $\begin{array}{l}\text { A part of the Laniakea Supercluster. It also } \\
\text { contains the Milky Way Galaxy, which contains } \\
\text { the Solar System. }\end{array}$ \\
\hline
\end{tabular}

WUM. The calculated maximum mass of the supercluster DM Core of $2.1 \times$ $10^{19}$ solar mass (see Table 1 ) is in good agreement with the values estimated in [18] and discussed in [21]. In the future, these stupendously large compact objects can give rise new Luminous Superclusters as the result of their DM Cores' Rotational Fission DOT 13.77 billion years ago, the estimated number of DM 
Supercluster Cores in the World was around $\sim 10^{3}$. It is unlikely that all of them gave birth to Luminous Superclusters at the same cosmological time being far away from each other. In our view, there were many "Beginnings" for different Luminous Superclusters (see Section 7).

In frames of WUM, Laniakea Supercluster emerged 13.77 billion years ago due to the Rotational Fission of Its overspinning DM Core and self-annihilation of DMPs (see Section 7). The Core was created during Dark Epoch when only Dark Matter Macroobjects existed [5]. The neighboring superclusters to LSC arise due to the Rotational Fission of their overspinning DM Cores and self-annihilation of DMPs, but in different times. In our view, the World consists of Multiworlds, which originated by different overspinning DM Cores of Superclusters at different times. The distribution of Macroobjects in the World is not only spatially Inhomogeneous and Anisotropic, but temporally non-simultaneous. Cosmological principal is valid only for the Homogeneous and Isotropic Medium that arised 14.22 Gyr ago. We emphasize that Time, Space, Gravity, Physical Laws are all emergent phenomena, which depend on the characteristics of the Medium only!

The mass-to-light ratio of the Virgo Supercluster (VS) is about 300 times larger than that of the Solar ratio. Similar ratios are obtained for other superclusters [22]. In 1933, F. Zwicky investigated the velocity dispersion of Coma cluster and found a surprisingly high mass-to-light ratio ( 500). He concluded: "If this would be confirmed, we would get the surprising result that dark matter is present in much greater amount than luminous matter" [23]. These ratios are one of the main arguments in favor of presence of large amounts of Dark Matter in the World.

Hubble tension is the disagreement in the values of the Hubble's constant $H_{0}$ obtained by the various teams. It can be explained the following way:

- All measurements of Hubble's constant are model-dependent;

- Statistics of these measurements is not sufficient to yield reliable conclusions;

- Observations of Galaxies belonging to different Superclusters;

- The experimental observations of galaxies in the universe show that most of them are disk galaxies [24]. It is well-known that when observing spiral galaxies, the side spinning toward us have a slight blueshift relative to the side spinning away from us. There is the meaning of a redshift of Centers of galaxies only;

- In LSC, some galaxies are moving toward MW, and some are moving away (see Figure 1). We can only meaningfully discuss a redshift of the Centre of LSC (0.0708).

According to WUM, the value of $H$ depends on the cosmological time: $H=\tau^{-1}$. It means that the value of $H$ should be measured based on Cosmic Microwave Background Radiation only. WUM calculates the value of the Hubble's constant $H_{0}=68.7494 \mathrm{~km} / \mathrm{s} \cdot \mathrm{Mpc}$ that is in excellent agreement with the most recent determinations using only Cosmic Microwave Background data: 
$H_{0}=68.7 \pm 1.3 \mathrm{~km} / \mathrm{s} \cdot \mathrm{Mpc}$ and $H_{0}=68.8 \pm 1.5 \mathrm{~km} / \mathrm{s} \cdot \mathrm{Mpc}$ [25].

\section{Volcanic Rotational Fission}

Lunar Origin Fission Hypothesis was proposed by G. Darwin in 1879 to explain the origin of Moon by fast spinning Earth, on which equatorial gravitative attraction was nearly overcome by centrifugal force [26].

Solar Fission Theory was proposed by L. Jacot in 1951 who stated that [27]:

- The planets were expelled from the Sun one by one from the equatorial bulge caused by rotation;

- One of these planets shattered to form the asteroid belt,

- Moons and rings of planets were formed from the similar expulsion of material from their parent planets.

T. Van Flandern further extended this theory in 1993 [28]. He proposed that planets were expelled from the Sun in pairs at different times. Six original planets exploded to form the rest of the modern planets. It solves several problems the standard Nebular Hypothesis does not:

- If planets fission from the Sun due to overspin while the proto-Sun is still accreting, this more easily explains how $98 \%$ of the Solar system's angular momentum ended up in the planets;

- It solves the mystery of the dominance of prograde rotation for these original planets since they would have shared in the Sun's prograde rotation at the outset,

- It also explains coplanar and circular orbits,

- It is the only model that explains the twinning of planets (and moons) and difference of planet pairs because after each planet pair is formed in this way, it will be some time before the Sun and extended cloud reach another overspin condition.

The outstanding issues of the Solar fission are:

- It is usually objected that tidal friction between a proto-planet and a gaseous parent, such as the proto-Sun, ought to be negligible because the gaseous parent can reshape itself so that any tidal bulge has no lag or lead, and therefore transfers no angular momentum to the proto-planet,

- There would exist no energy source to allow for planetary explosions.

Neither L. Jacot nor T. Van Flandern proposed an origin for the Sun itself. It seems that they followed the standard Nebular hypothesis of the formation of the Sun. In WUM, we concentrated on furthering the Solar fission theory [29].

Angular Momentum Problem is one of the most critical problem in Standard Cosmology that must be solved. Standard Cosmology does not explain how Galaxies and Extrasolar systems obtained their enormous orbital angular momenta [30]:

- Solar System (SS) has an orbital momentum $L_{\text {orb }}^{S S}$ calculated based on the distance of $26.4 \mathrm{kly}$ from the galactic Centre and orbital speed of about 220 $\mathrm{km} / \mathrm{s}: \quad L_{\text {orb }}^{S S}=1.1 \times 10^{56} \mathrm{~J} \cdot \mathrm{s}$, which far exceeds the rotational angular momen- 
tum: $\quad L_{\text {rot }}^{S S}=3.2 \times 10^{43} \mathrm{~J} \cdot \mathrm{s}$;

- Milky Way (MW) galaxy is gravitationally bounded with the Virgo Supercluster and has an orbital angular momentum $L_{\text {orb }}^{M W}$ calculated based on the distance of 65 million light-years from Virgo Supercluster and orbital speed of about $400 \mathrm{~km} / \mathrm{s}[31]: L_{\text {orb }}^{M W}=2.5 \times 10^{71} \mathrm{~J} \cdot \mathrm{s}$, which far exceeds the rotational angular momentum of MW [30]: $L_{\text {rot }}^{M W} \approx 1 \times 10^{67} \mathrm{~J} \cdot \mathrm{s}$.

In our opinion, there is the only one mechanism that can supply angular momenta to Macroobjects-Rotational Fission of overspinning (surface speed at equator exceeding escape velocity) Prime Objects. From the point of view of Fission model, the Prime Object is transferring some of its rotational angular momentum to orbital and rotational momenta of satellites. It follows that the rotational momentum of the prime object should exceed the orbital momentum of its satellite.

In frames of WUM, Prime Objects are Dark Matter (DM) Cores of Superclusters, which must accumulate tremendous rotational angular momenta before the Birth of the Luminous World. It means that it must be some long enough time in the history of the World, which we named "Dark Epoch" [5]. To be consistent with the Law of Conservation of Angular Momentum we developed a New Cosmology of the World:

- WUM introduces Dark Epoch (spanning from the Beginning of the World for 0.45 billion years) when only DM Macroobjects (MOs) existed, and Luminous Epoch (ever since for 13.77 billion years) when Luminous MOs emerged due to the Rotational Fission of Overspinning DM Superclusters' Cores and self-annihilation of DMPs;

- Proposed Weak Interaction of DMPs with Matter (DM and Baryonic Matter, see below) provides the integrity of DM Cores, which are 3D fluid balls with a high viscosity and act as solid-state objects;

- The main objects of the World are overspinning DM Cores of Superclusters, which accumulated tremendous rotational angular momenta during Dark Epoch and transferred it to DM Cores of Galaxies during their Rotational Fission. The experimental observations of galaxies in the universe showed that most of them are disk galaxies: about $60 \%$ are ellipticals and about $20 \%$ are spirals [24]. These results speak in favor of the developed Rotational Fission mechanism;

- Size, mass, density, composition, $L_{\text {orb }}$ and $L_{\text {rot }}$ of satellite cores depend on local density fluctuations at the edge of the overspinning prime DM cores and cohesion of the outer shell. Consequently, the diversity of satellite cores has a clear explanation;

- In our view, satellite DM cores are given off by "Volcanoes" on prime DM cores erupting repeatedly over millions or billions of years;

- Dark Matter Core of MW was born 13.77 billion years ago as the result of the Volcanic Rotational Fission of the Virgo Supercluster DM Core;

- DM Cores of Extrasolar systems, planets and moons were born as the result 
of the repeating Volcanic Rotational Fissions of MW DM Core in different times ( 4.57 billion years ago for SS);

- Macrostructures of the World form from the top (superclusters) down to galaxies, extrasolar systems, planets, and moons;

- Macrostructures of the World form from the top (superclusters) down to galaxies, extrasolar systems, planets, and moons.

Weak Interaction. A widely discussed models for nonbaryonic DM are based on the Cold DM hypothesis, and corresponding particles are commonly assumed to be WIMPs, which interact via gravity and any other force (or forces), potentially not part of the standard model itself, which is as weak as or weaker than the weak nuclear force, but also non-vanishing in its strength [32]. It follows that a new weak force needs to exist, providing interaction between DMPs. The strength of this force exceeds that of gravity, and its range is considerably greater than that of the weak nuclear force [5].

According to WUM, strength of gravity is characterized by the gravitational parameter [1]:

$$
G=G_{0} \times Q^{-1}
$$

where $G_{0}=a^{2} c^{4} / 8 \pi h c$ is an extrapolated value of $G$ at the Beginning of the World $(Q=1)$. The range of the gravity equals to the size of the World $R$ :

$$
R=a \times Q=1.34558 \times 10^{26} \mathrm{~m}
$$

In WUM, weak interaction is characterized by the parameter $G_{W}$ :

$$
G_{W}=G_{0} \times Q^{-1 / 4}
$$

which is about 30 orders of magnitude greater than $G$. The range of the weak interaction $R_{W}$ in the present Epoch equals to:

$$
R_{W}=a \times Q^{1 / 4}=1.65314 \times 10^{-4} \mathrm{~m}
$$

that is much greater than the range of the weak nuclear force. Calculated concentration of DMF4 $n_{D M F 4}$ in the largest shell of Superclusters: $n_{D M F 4} \cong 4.2 \times 10^{15} \mathrm{~m}^{-3}$ shows that a distance between particles is around $\sim 10^{-5} \mathrm{~m}$, which is much smaller than $R_{W}$. Thus, the introduced weak interaction of DMPs with Matter will provide integrity of all Shells. In our view, weak interaction between particles DMF3 provides integrity of Fermi Bubbles and Solar Corona [33].

Based on the proposed New Cosmology, we performed a detailed analysis of the angular momenta of main objects of the World-overspinning DM Cores of superclusters and galaxies. According to the WUM theory of Compact Objects, parameters of Macroobjects Cores made up of different DMFs in Dark Epoch $(0.45 \mathrm{Gyr})$ before Rotational Fission are as follows $\left(m_{0}\right.$ is a basic unit of mass $\left.m_{0}=h / a c\right)$ [29]:

Supercluster DM Core (based on DMF4):

- Maximum mass $M_{0.45}^{S C}$ equals to: $M_{0.45}^{S C}=\frac{4 \pi}{3} m_{0} \alpha^{-8} \times Q_{0.45}^{3 / 2}=2.4 \times 10^{47} \mathrm{~kg}$;

- Minimum radius $R_{0.45}^{S C}$ equals to: $R_{0.45}^{S C}=2 \pi a \alpha^{-8} \times Q_{0.45}^{1 / 2}=3.4 \times 10^{22} \mathrm{~m}$; 
- Rotational angular momentum $L_{0.45}^{S C}$ equals to: $L_{0.45}^{S C}=4.7 \times 10^{77} \mathrm{~J} \cdot \mathrm{s}$. Galaxy DM Core (based on DMF3);

- Maximum mass $M_{0.45}^{G C}$ equals to: $M_{0.45}^{G C}=\frac{4 \pi}{3} m_{0} \alpha^{-4} \times Q_{0.45}^{3 / 2}=6.8 \times 10^{38} \mathrm{~kg}$;

- Minimum radius $R_{0.45}^{G C}$ equals to: $R_{0.45}^{G C}=2 \pi a \alpha^{-4} \times Q_{0.45}^{1 / 2}=9.6 \times 10^{13} \mathrm{~m}$;

- Rotational angular momentum $L_{0.45}^{G C}$ equals to: $L_{0.45}^{G C}=3.2 \times 10^{60} \mathrm{~J} \cdot \mathrm{s}$.

Milky Way (MW) is gravitationally bounded with Virgo Supercluster (VS) [31]. Let us compare $L_{0.45}^{V S}$ with an orbital momentum of Milky Way $L_{\text {orb }}^{M W}$ calculated based on the distance of 65 million light years from VS and orbital speed of about $400 \mathrm{~km} / \mathrm{s}[31]: L_{o r b}^{M W}=2.5 \times 10^{71} \mathrm{~J} \cdot \mathrm{s}$. It follows that as the result of rotational Fission of VS Core, approximately $\sim 10^{6}$ galaxies like MW could be generated at the same time. Considering that the number density of galaxies in the VS falls off with the square of the distance from its center and the location of MW on the outskirts of the VS [34], the actual number of created galaxies could be much larger.

Comparison of the SS orbital momentum $L_{\text {orb }}^{S S}=1.1 \times 10^{56} \mathrm{~J} \cdot \mathrm{s}$ with rotational momentum of MW galaxy DM Core $L_{0.45}^{G C}=3.2 \times 10^{60} \mathrm{~J} \cdot \mathrm{s}$ shows that approximately $\sim 10^{4}$ Extrasolar systems like SS could be created at the same time. Considering that MW has grown inside out (in the present Epoch, most old stars can be found in the middle, more recently formed ones on the outskirts [35]), the number of generated Extrasolar systems could be much larger. Extrasolar system DM Cores can give birth to planet DM cores, and they can generate DM cores of moons by the same Volcanic Rotational Fission mechanism.

The calculated value of the total mass of DM Macroobjects in Dark Epoch $M_{0.45}^{M O}$ before Rotational Fission is: $M_{0.45}^{M O}=2 \pi^{2} m_{0} \times Q_{0.45}^{2}$ and the minimum number of DM superclusters is: $N_{0.45}^{S C}=1.5 \pi \alpha^{8} \times Q_{0.45}^{1 / 2} \sim 3 \times 10^{3}$. It is unlikely that all of them gave birth to Luminous Superclusters at the same cosmological time being far away from each other. In our view, there were many "Beginnings" for different Luminous Superclusters. It is worth noting that the Absolute Age of the entire World is 14.22 Gyr. No one supercluster can be older than $14.22 \mathrm{Gyr}$.

\section{Dark Matter Reactors}

The following facts support the existence of Dark Matter Cores in Macroobjects [1]:

- E. Fossat, et al. found that Solar Core rotates $3.8 \pm 0.1$ faster than the surrounding envelope [36];

- J. Zhang, et al. concluded that the Earth's inner core is rotating faster than its surface by about 0.3 - 0.5 degrees per year [37];

- T. Guillot, et al. found that a deep interior of Jupiter rotates nearly as a rigid body, with differential rotation decreasing by at least an order of magnitude compared to the atmosphere [38];

- W. Wu, S. Ni, and J. Irving found that the Earth's $660-\mathrm{km}$ boundary is rougher than the surface layer that we all live on [39]; 
- Random variations of Earth's and Venus's Rotational Speed: the variations of the Earth daylength throughout 2020 were in the range $86400_{-1.46 \mathrm{~ms}}^{+1.62 \mathrm{ss}}$ [40] and the average sidereal day on Venus in the 2006-2020 interval was 243.0226 \pm 0.0013 Earth days [41];

- Plutonium-244 with half-life of 80.6 million years and Iron-60 with half-life of 2.6 million years are not produced in significant quantities by the nuclear fuel cycle, because it needs very high neutron flux environments [42]. Any Pu-244 and Iron-60 present in the Earth's crust should have decayed by now. Nevertheless, D. C. Hoffman, et al. in 1971 obtained the first indication of $\mathrm{Pu}-244$ present existence in the Nature [43] and A. Wallner, et al. in 2021 obtained signatures of Pu-244 and Iron-60 in samples of Pacific Ocean crust [42];

- Giant planets like Jupiter are hundreds of degrees warmer than current temperature models predict. Saturn radiates 2.5 times more energy than it receives from the Sun; Uranus-1.1 times; Neptune-2.6 times. Many Icy Solar system bodies including Pluto possess subsurface oceans.

According to WUM:

- The fact that Macroobject Cores rotate faster than surrounding envelopes, despite high viscosity of the internal medium, is intriguing. WUM explains this phenomenon through absorption of DMPs by Cores. Dark Matter Particles supply not only additional mass $\left(\propto \tau^{3 / 2}\right)$, but also additional angular momentum $\left(\propto \tau^{2}\right)$. Cores irradiate products of annihilation, which carry away excessive angular momentum. The Solar Wind is the result of this mechanism [5];

- The $660-\mathrm{km}$ boundary is a boundary between Earth's DM core and Upper mantle with Crust, which were produced by DM core during 4.57 billion years [31];

- Pu-244 and Iron-60 are produced within the Earth as the result of DMF1 particles self-annihilation. They arrive to the Crust of the Earth due to convection currents in the mantle carrying isotopes from the interior to the planet's surface [44];

- Random variations of the Earth's rotational speed on a daily basis can be explained by variations in an activity of the Earth's Dark Matter Reactor. As the result of DMPs self-annihilation, random mass ejections are happening. During a time of high DM Reactor activity, the Earth's rotational speed is lower (long days) due to increase of the Earth's moment of inertia. When random mass ejections are less frequent, the Earth's moment of inertia is decreasing, we observe short days.

- The internal heating of all gravitationally-rounded objects of the Solar system is due to DMPs self-annihilation in their cores made up of DMF1 $(1.3 \mathrm{TeV})$. The amount of energy produced due to this process is sufficiently high to heat up the objects. New DMF1 freely penetrate through the entire objects' envelope, get absorbed into the cores, and continuously support DMF1 
self-annihilation.

Macroobjects' cores are essentially Dark Matter Reactors fueled by DMPs. All chemical elements, gases, water vapors, compositions, radiations are produced by Macroobjects themselves as the result of DMPs self-annihilation. The diversity of all gravitationally-rounded Macroobjects in the Solar system is explained by the differences in their DM cores (mass, size, density, composition). The DM Reactors at their cores (including Earth) are very efficient and provide enough energy for the internal heating and all their geological processes like volcanos, quakes, mountains' formation through tectonic forces or volcanism, tectonic plates' movements, etc. All gravitationally-rounded Macroobjects in hydrostatic equilibrium, down to Mimas in Solar system, prove the validity of WUM.

\section{Early Earth}

Formation of Earth. The oldest material found in SS is dated to 4.568 Gyr ago [45]. In the article "The age of the Earth in the twentieth century: a problem (mostly) solved" G. B. Dalrymple said: Whether this age represents the age of the Earth's accretion, of core formation, or of the material from which the Earth formed is not yet known, but recent evidence suggests it may approximate the latter [46].

In WUM, DM core of the Earth with the radius of $R_{E}=3.52 \times 10^{3} \mathrm{~km}$ was born $4.57 \mathrm{Gyr}$ ago as the result of the Volcanic Rotational Fission of the Sun's DM Core with the radius of $R_{S}=487 \times 10^{3} \mathrm{~km}$ [47].

Origin of the Moon is usually explained by a Mars-sized body striking the Earth, making a debris ring that eventually collected into a single natural satellite, the Moon, but there are a number of variations on this giant-impact hypothesis, as well as alternative explanations, and research continues into how the Moon came to be. Other proposed scenarios include captured body, fission, formed together (condensation theory, Synestia), planetesimal collisions (formed from asteroid-like bodies), and collision theories. The standard giant-impact hypothesis suggests that a Mars-sized body, called Theia, impacted the proto-Earth, creating a large debris ring around Earth, which then accreted to form the Moon [48].

Establishing the age of the Moon is critical to understanding SS evolution and the formation of rocky planets, including Earth. However, despite its importance, the age of the Moon has never been accurately determined before 2017 . M. Barboni, et al. "present uranium-lead dating of Apollo 14 zircon fragments that yield highly precise, concordant ages, demonstrating that they are robust against postcrystallization isotopic disturbances. Hafnium isotopic analyses of the same fragments show extremely low initial ${ }^{176} \mathrm{Hf}^{177} \mathrm{Hf}$ ratios corrected for cosmic ray exposure that are near the solar system initial value. Our data indicate differentiation of the lunar crust by 4.51 billion years, indicating the formation of the Moon within the first $\sim 60$ million years after the birth of the solar system" [49]. 
Following the prevailing giant-impact hypothesis, planetary geophysicists at the German Aerospace Center, led by M. Maurice, have used a new numerical model to reconstruct the time at which the event occurred. They report that the Moon formed $4.425 \pm 0.025$ billion years ago, and that it hosted an ocean of magma for substantially longer time than previously thought (for $~ 200$ million years) $[50]$.

In WUM, DM core of the Moon with the radius of $R_{M}=0.381 \times 10^{3} \mathrm{~km}$ was born as the result of the Volcanic Rotational Fission of the Earth's DM Core $\lesssim$ 4.57 Gyr [47].

Continental Crust of Earth. The long-favored paradigm for the development of continental crust is one of progressive growth beginning at $\sim 4$ billion years ago. To test this hypothesis, T. M. Harrison, et al. measured initial ${ }^{176} \mathrm{Hf} /{ }^{177} \mathrm{Hf}$ values of 4.01 - 4.37 Gyr detrital zircons from Western Australia. They obtained results that support the view that crust had formed by $4.4-4.5 \mathrm{Gyr}$ and was rapidly recycled into the mantle [51].

Earth's Atmosphere and Oceans were formed by volcanic activity and outgassing. Most of the gas was carbon dioxide and water vapor that condensed into oceans. In this model, atmospheric greenhouse gases kept the oceans from freezing when the newly forming Sun had only $70 \%$ of its current luminosity.

According to the "Lumen Learning Earth Science" [52]: Scientists have developed a number of hypotheses about how the oceans formed. Though these hypotheses have changed over time, one idea now has the wide support of Earth scientists, called the volcanic outgassing theory. This means that water vapor given off by volcanoes erupting over millions or billions of years, cooled and condensed to form Earth's oceans.

According to the "National Ocean Service" [53]: Most scientists agree that the atmosphere and the ocean accumulated gradually over millions and millions of years with the continual 'degassing' of the Earth's interior. According to this theory, the ocean formed from the escape of water vapor and other gases from the molten rocks of the Earth to the atmosphere surrounding the cooling planet. After the Earth's surface had cooled to a temperature below the boiling point of water, rain began to fall-and continued to fall for centuries. As the water drained into the great hollows in the Earth's surface, the primeval ocean came into existence. The forces of gravity prevented the water from leaving the planet.

In the paper "Uncovering Mysteries of Earth's Primeval Atmosphere 4.5 Billion Years Ago and the Emergence of Life" ETH Zurich (a leading scientist P. Sossi) wrote [54]: Four-and-a-half billion years ago, Earth would have been hard to recognize. Instead of the forests, mountains, and oceans that we know today, the surface of our planet was covered entirely by magma-the molten rocky material that emerges when volcanoes erupt. This much the scientific community agrees on. What is less clear is what the atmosphere at the time was like.

In the paper "Redox state of Earth's magma ocean and its Venus-like early atmosphere" [55], P. A. Sossi, et al. found that after cooling down from the 
magma state, the young Earth had an atmosphere that was slightly oxidizing, with carbon dioxide as its main constituent, as well as nitrogen and some water. The surface pressure was also much higher, almost one hundred times that of today and the temperature was much higher, due to the hot surface. These characteristics made it more similar to the atmosphere of today's Venus than to that of today's Earth. Based on their results, the authors made a conclusion that a popular theory on the emergence of life on Earth, in which lightning strikes interact with certain gases (notably ammonia and methane) to create amino acids-the building blocks of life-seems much less likely. The necessary gases were simply not sufficiently abundant.

Origin of Life. M. Dodd, et al. in the article "Evidence for early life in Earth's oldest hydrothermal vent precipitates" wrote [56]: Although it is not known when or where life on Earth began, some of the earliest habitable environments may have been submarine-hydrothermal vents. Here we describe putative fossilized microorganisms that are at least 3770 million and possibly 4280 million years old in ferruginous sedimentary rocks, interpreted as seafloor-hydrothermal vent-related precipitates. These structures occur as micrometre-scale haematite tubes and filaments with morphologies and mineral assemblages similar to those of filamentous microorganisms from modern hydrothermal vent precipitates and analogous microfossils in younger rocks. Collectively, these observations are consistent with an oxidized biomass and provide evidence for biological activity in submarine-hydrothermal environments more than 3770 million years ago [54].

The proposed concept of Dark Matter Reactors in Cores of all gravitationally-rounded Macroobjects successfully explains all these hypothesis and results for the Early Earth:

- The Upper mantle with Crust are due to the DM core volcanic activity of the "homemade" compositions (including magma), which produced as the result of the self-annihilation of DMPs in the DM core. It explains the result that continental crust had formed by $4.4-4.5 \mathrm{Gyr}$;

- Earth's Atmosphere and Oceans were formed by the volcanic activity and outgassing of DM core;

- The thickness of the Upper mantle with Crust is growing in time: the Early Earth had a smaller thickness than it is in the present time. Hence, the temperature of the Earth's surface was higher than its calculated temperature based on the Sun's output at that time. It kept the oceans from freezing when the newly forming Sun had only 70\% of its current luminosity;

- The biological activity in submarine-hydrothermal environments more than 3770 million years ago can be explained by a generation of all kinds of chemical elements and compositions produced into the Earth's DM core.

\section{Conclusion}

WUM does not attempt to explain all available cosmological and geophysical 
data, as that is an impossible feat for any one article. Nor does WUM pretend to have built an all-encompassing theory that can be accepted as is. The Model needs significant further elaboration, but in its present shape, it can already serve as a basis for a new Cosmology proposed by Paul Dirac in 1937 and a new Geophysics. The Model should be developed into the well-elaborated theory by the entire physical community.

\section{Acknowledgements}

Special thanks to my son Ilya Netchitailo who helped me refine the Model and improve its understanding.

\section{Conflicts of Interest}

The author declares no conflicts of interest regarding the publication of this paper.

\section{References}

[1] Netchitailo, V.S. (2021) Paradigm Shift in Cosmology. https://vixra.org/pdf/2107.0020v2.pdf

[2] Netchitailo, V. (2018) Hypersphere World-Universe Model. Tribute to Classical Physics. Journal of High Energy Physics, Gravitation and Cosmology, 4, 441-470. https://doi.org/10.4236/jhepgc.2018.43024

[3] Tesla, N. (1937) Prepared Statement on the 81st Birthday Observance. http://www.institutotesla.org/tech/TeslaGravity.html

[4] Dirac, P.M. (1951) Is There an Æther? Nature, 168, 906-907. https://web.archive.org/web/20081217042934/http://dbhs.wvusd.k12.ca.us/webdocs /Chem-History/Planck-1901/Planck-1901.html https://doi.org/10.1038/168906a0

[5] Netchitailo, V. (2019) Dark Matter Cosmology and Astrophysics. Journal of High Energy Physics, Gravitation and Cosmology, 5, 999-1050. https://doi.org/10.4236/jhepgc.2019.54056

[6] Netchitailo, V. (2017) Mathematical Overview of Hypersphere World-Universe Model. Journal of High Energy Physics, Gravitation and Cosmology, 3, 415-437. https://doi.org/10.4236/jhepgc.2017.33033

[7] Netchitailo, V. (2020) World-Universe Model Predictions. Journal of High Energy Physics, Gravitation and Cosmology, 6, 282-297. https://doi.org/10.4236/jhepgc.2020.62022

[8] Li, Q., Xue, C., Liu, J.-P., Wu, J.-F., Yang, S.-Q., Shao, C.-G., et al. (2018) Measurements of the Gravitational Constant Using Two Independent Methods. Nature, 560 582-588. https://doi.org/10.1038/s41586-018-0431-5

[9] Fixsen, D.J. (2009) The Temperature of the Cosmic Microwave Background. Astrophysical Journal, 707, 916-920. https://doi.org/10.1088/0004-637X/707/2/916

[10] Netchitailo, V.S. (2013) Word-Universe Model. viXra:1303.0077v7. https://vixra.org/pdf/1303.0077v7.pdf

[11] Hoyle, F. and Narlikar, J.V. (1964) A New Theory of Gravitation. Proceedings of the Royal Society of London, A282, 191-207. https://doi.org/10.1098/rspa.1964.0227

[12] Dirac, P.A.M. (1974) Cosmological Models and the Large Numbers Hypothesis. 
Proceedings of the Royal Society of London, A338, 439-446. https://doi.org/10.1098/rspa.1974.0095

[13] Riemann, B. (1854) On the Hypotheses Which Lie at the Bases of Geometry. Translated by William Kingdon Clifford. Nature, 8, 36-37. https://doi.org/10.1038/008036a0

[14] Netchitailo V.S. (2020) Hypersphere World-Universe Model Cosmological Time. viXra:2011.0038. https://vixra.org/pdf/2011.0038v1.pdf

[15] Boehm, C., Fayet, P. and Silk, J. (2003) Light and Heavy Dark Matter Particles. Physical Review D, 69, Article ID: 101302. https://doi.org/10.1103/PhysRevD.69.101302

[16] Netchitailo, V. (2017) Astrophysics: Macroobject Shell Model. Journal of High Energy Physics, Gravitation and Cosmology, 3, 776-790. https://doi.org/10.4236/jhepgc.2017.34057

[17] Mehrgan, K., Thomas, J., Saglia, R., Mazzalay, X., Erwin, P., Bender, R., et al. (2019) A 40-Billion Solar Mass Black Hole in the Extreme Core of Holm 15A, the Central Galaxy of Abell 85. The Astrophysical Journal, 887, Article No. 195. https://doi.org/10.3847/1538-4357/ab5856

[18] Shemmer, O., Netzer, H., Maiolino, R., Oliva, E., Croom, S., Corbett, E.A., et al. (2004) Near-Infrared Spectroscopy of High Redshift Active Galactic Nuclei. I. A Metallicity-Accretion Rate Relationship. The Astrophysical Journal, 614, 547-557. https://doi.org/10.1086/423607

[19] Brent Tully, R., Courtois, H., Hoffman, Y. and Pomarède, D. (2014) The Laniakea Supercluster of Galaxies. Nature, 513, 71-73. https://doi.org/10.1038/nature13674

[20] Wikipedia (n.d.) List of Largest Cosmic Structures. https://en.wikipedia.org/wiki/List_of_largest_cosmic_structures

[21] Carr, B., Kühnel, F. and Visinelli, L. (2021) Constraints on Stupendously Large Black Holes. Monthly Notices of the Royal Astronomical Society, 501, 2029-2043. https://doi.org/10.1093/mnras/staa3651

[22] Heymans, C., Gray, M.E., Peng, C.Y., Van Waerbeke, L., Bell, E.F., Wolf, C., et al. (2008) The Dark Matter Environment of the Abell 901/902 Supercluster: A Weak Lensing Analysis of the HST STAGES Survey. Monthly Notices of the Royal Astronomical Society, 385, 1431-1442.

[23] Zwicky, F. (1933) Die Rotverschiebung von extragalaktischen Nebeln. Helvetica Physica Acta, 6, 110.

[24] Cool Cosmos (2021) What Are Galaxies? https://coolcosmos.ipac.caltech.edu/ask/216-What-are-galaxies-

[25] NASA Education/Graphics (2021) Hubble Constant $\mathrm{H}_{0}$. https://lambda.gsfc.nasa.gov/education/graphic_history/hubb_const.cfm

[26] Darwin, G.H. (1879) On the Bodily Tides of Viscous and Semi-Elastic Spheroids, and on the Ocean Tides upon a Yielding Nucleus. Philosophical Transactions of the Royal Society of London, 170, 1-35. https://doi.org/10.1098/rstl.1879.0061

[27] Jacot, L. (1986) Heretical Cosmology: The Catastrophic Dislocations of Galaxies, Stars and Planets. Exposition-Banner of Florida.

https://www.amazon.com/Heretical-Cosmology-Catastrophic-Dislocations-Expositi on-Banner/dp/0682401757

[28] Van Flandern, T. (1999) Dark Matter, Missing Planets, and New Comets. North Atlantic.

https://www.amazon.com/Dark-Matter-Missing-Planets-Comets/dp/1556432682 
[29] Netchitailo, V. (2016) Overview of Hypersphere World-Universe Model. Journal of High Energy Physics, Gravitation and Cosmology, 2, 593-632. https://doi.org/10.4236/jhepgc.2016.24052

[30] Netchitailo, V. (2019) Solar System. Angular Momentum. New Physics. Journal of High Energy Physics, Gravitation and Cosmology, 5, 112-139. https://doi.org/10.4236/jhepgc.2019.51005

[31] NASA (National Aeronautics and Space Administration) (2015) The Cosmic Distance Scale.

https://imagine.gsfc.nasa.gov/features/cosmic/local_supercluster_info.html

[32] Wikipedia (n.d.) Weakly Interacting Massive Particles. https://en.wikipedia.org/wiki/Weakly_interacting_massive_particles

[33] Netchitailo, V. (2021) Hypersphere World-Universe Model. Journal of High Energy Physics, Gravitation and Cosmology, 7, 915-941. https://doi.org/10.4236/jhepgc.2021.72042

[34] Tully, R.B. (1982) The Local Supercluster. Astrophysical Journal, 257, 389-422. https://doi.org/10.1086/159999

[35] Ness, M., Hogg, D.W., Rix, H.-W., Ho, A.Y.Q. and Zasowski, G. (2015) The Cannon: A Data-Driven Approach to Stellar Label Determination. The Astrophysical Journal, 808, Article No. 16. https://doi.org/10.1088/0004-637X/808/1/16

[36] Fossat, E., Boumier, P., Corbard, T., Provost, J., Salabert, D., Schmider, F.X., et al. (2017) Asymptotic G Modes: Evidence for a Rapid Rotation of the Solar Core. Astronomy \& Astrophysics, 604, Article No. A40. https://doi.org/10.1051/0004-6361/201730460

[37] Zhang, J., Song, X., Li, Y., Richards, P.G., Sun, X. and Waldhauser, F. (2005) Inner Core Differential Motion Confirmed by Earthquake Waveform Doublets. Science, 309, 1357-1360. https://doi.org/10.1126/science.1113193

[38] Guillot, T., Miguel, Y., Militzer, B., Hubbard, W.B., Kaspi, Y., Galanti, E., et al. (2018) A Suppression of Differential Rotation in Jupiter's Deep Interior. Nature, 555, 227-230. https://www.nature.com/articles/nature25775

[39] Wu, W., Ni, S. and Irving, J.C.E. (2019) Inferring Earth's Discontinuous Chemical Layering from the 660-Kilometer Boundary Topography. Science, 363, 736-740.

[40] Jones, G. and Bikos K. (2020) Earth Is in a Hurry in 2020. https://www.timeanddate.com/time/earth-faster-rotation.html

[41] Margot, J.-L., Campbell, D.B., Giorgini, J.D., Jao, J.S., Snedeker, L.G., Ghigo, F.D., et al. (2021) Spin State and Moment of Inertia of Venus. Nature Astronomy, 5, 676-683. https://doi.org/10.1038/s41550-021-01339-7

[42] Wallner, A., Froehlich, M.B., Hotchkis, M.A.C., Kinoshita, N., Paul, M., et al. (2021) ${ }^{60} \mathrm{Fe}$ and ${ }^{244} \mathrm{Pu}$ Deposited on Earth Constrain the R-Process Yields of Recent Nearby Supernovae. Science, 372, 742-745. https://doi.org/10.1126/science.aax3972 https://www.science.org/doi/10.1126/science.aax3972

[43] Hoffman, D.C., Lawrence, F.O., Mewherter, J.L. and Rourke, F.M. (1971) Detection of Plutonium-244 in Nature. Nature, 234, 132-134. https://doi.org/10.1038/234132a0

[44] Bercovici, D. (2010) Treatise on Geophysics, Volume 7: Mantle Dynamics. Elsevier, Science. https://books.google.com/books?id=bIHNCgAAQBAJ

[45] Bouvier, A. and Wadhwa, M. (2010) The Age of the Solar System Redefined by the Oldest $\mathrm{Pb}-\mathrm{Pb}$ Age of a Meteoritic Inclusion. Nature Geoscience, 3, 637-641. https://doi.org/10.1038/ngeo941 
[46] Dalrymple, G.B. (2001) The Age of the Earth in the Twentieth Century: A Problem (Mostly) Solved. Geological Society, London, Special Publications, 190, 205-221. https://doi.org/10.1144/GSL.SP.2001.190.01.14

[47] Netchitailo, V.S. (2021) Solar System. Angular Momentum. Dark Matter Reactors. https://vixra.org/pdf/2108.0107v1.pdf

[48] Wikipedia (n.d.) Origin of the Moon. https://en.wikipedia.org/wiki/Origin_of_the_Moon\#cite_note-themoon-35

[49] Barboni, M., Boehnke, P., Keller, B., Kohl, I.E., Schoene, B., Young, E.D., et al. (2017) Early Formation of the Moon 4.51 Billion Years Ago. Science Advances, 3, Article ID: e1602365. https://doi.org/10.1126/sciadv.1602365

[50] Maurice, M., Tosi, N., Schwinger, S., Breuer, D. and Kleine, T. (2020) A Long-Lived Magma Ocean on a Young Moon. Science Advances, 6, Article ID: eaba8949. https://doi.org/10.1126/sciadv.aba8949

[51] Harrison, T.M., Blichert-Toft, J., Müller, W., Albarede, F., Holden, P. and Mojzsis, S.J. (2005) Heterogeneous Hadean Hafnium: Evidence of Continental Crust at 4.4 to 4.5 Ga. Science, 310, 1947-1950. https://doi.org/10.1126/science.1117926

[52] Earth Science (2021) Introduction to the Oceans. Lumen Learning. https://courses.lumenlearning.com/earthscience/chapter/introduction-to-the-ocean s/

[53] National Ocean Service (2021) Why Do We Have an Ocean? Administration National Ocean Service. https://oceanservice.noaa.gov/facts/why_oceans.html

[54] ETH Zurich (2020) Uncovering Mysteries of Earth's Primeval Atmosphere 4.5 Billion Years Ago and the Emergence of Life.

https://scitechdaily.com/uncovering-mysteries-of-earths-primeval-atmosphere-4-5billion-years-ago-and-the-emergence-of-life/

[55] Sossi, P.A., Burnham, A.D., Badro, J., Lanzirotti, A., Newville, M. and O’Neill, H.St.C. (2020) Redox State of Earth's Magma Ocean and Its Venus-Like Early Atmosphere. Science Advances, 6, Article ID: eabd1387. https://doi.org/10.1126/sciadv.abd1387

[56] Dodd, M., Papineau, D., Grenne, T., Slack, J.F., Rittner, M., Pirajno, F., et al. (2017) Evidence for Early Life in Earth's Oldest Hydrothermal Vent Precipitates. Nature, 543, 60-64. https://doi.org/10.1038/nature21377 